\title{
Neonatal mortality at the neonatal unit: the situation at a teaching hospital in Ghana
}

\author{
Benjamin Atta Owusu ${ }^{1}$, Apiradee Lim ${ }^{1}$, Nifatamah Makaje ${ }^{1}$, Priscilla Wobil ${ }^{2}$, Areeyuth SameAe ${ }^{1}$
}

\author{
1. Department of Mathematics and Computer Science, Faculty of Science and Technology, \\ Prince of Songkla University, Pattani, Thailand \\ 2. Child Health Directorate, Komfo Anokye Teaching Hospital, Kumasi, Ghana.
}

\begin{abstract}
Background: The first 28 days of life- the neonatal period is the most vulnerable time for a child's survival. Globally, neonatal mortality has seen a downward trend in recent years. The main objective of this study was to determine the percentage of neonatal mortality and to provide information on factors associated with neonatal mortality at the neonatal unit of a tertiary health facility or teaching hospital.

Methods: Data of neonates admitted to the neonatal in-patient unit of the Komfo Anokye Teaching Hospital (KATH) in Ghana from January 2013 to May 2014 were analyzed. Logistic regression model was performed to assess the association between neonatal mortality and predictors.

Results: A total of 5,195 neonatal admissions were recorded. The overall percentage of neonatal mortality was $20.2 \%$. Infants with very low birth weight, having 5-minute Apgar score lower than 4, newborns with pre-term delivery, being referred from other health facilities, and being diagnosed with respiratory distress and birth asphyxia had a higher percentage of neonatal mortality.

Conclusion: The mortality at the neonatal in-patient unit at the Komfo Anokye Teaching Hospital in Ghana is very high. There is the need for continuous attention and interventions to help reduce the risk of mortality among neonates admitted to the facility.
\end{abstract}

Keywords: Logistic regression, neonatal mortality, Kumasi.

DOI: https://dx.doi.org/10.4314/ahs.v18i2.22

Cite as: Owusu BA, Lim A, Makaje N, Wobil P, SameAe A. Neonatal mortality at the neonatal unit: the situation at a teaching hospital in Ghana. Afri Health Sci. 2018;18(2): 369-377. https:// dx.doi.org/10.4314/abs.v18i2.22

\section{Introduction}

The first four weeks of life are the most crucial period of life. It is within this period that infants are highly prone to illness and death. Each year, almost 3 million newborns die within the neonatal period with half of these deaths occurring within the first 24 hours of birth ${ }^{1,2}$. Most of these deaths are attributable to infections, pre-term delivery and birth asphyxia ${ }^{3}$. The current pace of reduc-

\section{Corresponding author:}

Benjamin Atta Owusu, Department of Mathematics and Computer Science, Faculty of Science and Technology,Prince of Songkla University, Pattani, Thailand.

Email: benteeta@gmail.com, 5720320702@email.psu.ac.th tion in neonatal deaths could be accelerated through the provision of feeding support, extra care for pre-term newborns, improving resuscitation skills and infection prevention ${ }^{4}$. These solutions are achievable, however, neonatal mortality remains a big challenge for lower-middle income countries, especially in sub-Saharan Africa and Southern Asia with more than two-third of neonatal mortality occur in these regions ${ }^{5}$. Countries in these two regions have made the least progress towards reducing neonatal mortality ${ }^{6}$.

In Ghana, neonatal mortality rate was found at 39 per 1000 live births in $2012^{19}$ and decreased to 30 per 1000 live births in 2014. However, neonatal mortality in Ghana remains higher than many other countries in Africa for example Ethiopia, Niger, Kenya, Malawi and Congo ${ }^{8}$. Thus, Ghana's Ministry of Health has launched the Ghana National Newborn Health Strategy and Action Plan

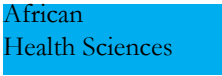

(C) 2018 Owusu et al. Licensee African Health Sciences. This is an Open Access article distributed under the terms of the Creative commons Attribution License (https://creativecommons.org/licenses/by/4.0), which permits unrestricted use, distribution, and reproduction in any medium, provided the original work is properly cited. 
2014-2018 to reduce the neonatal mortality rate to 21 per 1000 live births 7 . Separate studies have identified place of birth, birth weight, mode of delivery, delayed breastfeeding initiation and age of baby at the time of admission as significant predictors of neonatal death ${ }^{6}$. Examining the risk factors of neonatal mortality at the various neonatal units is necessary as it allows inferences about the quality of care. It could provide insights into how neonates could be managed to improve the outcomes of admissions at the neonatal unit.

The neonatal unit at the Komfo Anokye Teaching Hospital (KATH), serves as the main referral destination for sick newborns from almost six out of the ten regions in Ghana. The unit is equipped with advanced life-saving equipment to support these sick newborns.

The aim of this study was to investigate the major causes and risk factors of neonatal mortality and estimate the percentage of neonatal mortality based on the number of newborns admitted to the neonatal unit, also called Mother and Baby Unit (MBU), KATH, Kumasi, Ghana.

\section{Methods}

This was a hospital based study which used retrospective data from the neonatal in-patient unit to identify the major predictors of neonatal death at the hospital. This study was conducted at the MBU which is the neonatal in-patient unit of the KATH from January 2013 to May 2014. The KATH is a tertiary health facility located in a Kumasi, the capital of the Ashanti region and the most second most populous city in Ghana. KATH serves as the main referral facility for the Northern Sector of the country. The neonatal unit consists of three wards: a High Dependency Unit (HDU) that admits sick newborns referred from the delivery wards. There is also a pre-term or low birth weight (LBW) unit that admits pre-term or LBW newborns from the HDU who have been stabilized and a Septic unit that admits out-born newborns. Different categories of newborns are admitted to the facility. These include preterm newborns, low birth weight newborns, newborns with neonatal jaundice and sepsis and newborns with congenital anomalies. All neonates admitted to the unit from January 2013 to May 2014 were included in the study. These admissions included newborns who were delivered at different facilities and were referred to the unit on health grounds. The following information was retrieved from the in-patient treatment books: birth weight, sex, mode of delivery, place of delivery, age at admission, Apgar scores, time between birth and admission and outcome of admission. Information from 5,363 in-patient books was retrieved for the study. Out of this, 124 newborns were older than 28 days, hence were excluded from the study. Due to substantial missing information, 44 newborns were also excluded from the study. The newborns were grouped according to the place of delivery (whether they were delivered at KATH or referred from other facilities). Neonatal mortality was defined as death that occurred within the first 28 days of life.

The outcome of this study was neonatal mortality. It was defined as the number of neonates who died at the unit over the period of the study. This definition is found appropriate because all newborns at the neonatal unit are sick newborns brought in from either the hospital's delivery rooms or outside the hospital. Birth weights, mode of delivery, 5 minute Apgar score, gestational age, main diagnosis, gender and newborns' age at the time of admission were employed as exposure variables in this study. The birth weight was categorized into three groups namely; very low birth weight (VLBW), low birth weight (LBW), and normal weight. VLBW was defined as weight less than $1.5 \mathrm{~kg}$; LBW for those between $1.5-2.4 \mathrm{~kg}$, normal birth weight for those above $2.4 \mathrm{~kg}$. The mode of delivery was divided into three categories. The first group is spontaneous vaginal delivery (SVD) which includes all neonates delivered by spontaneous delivery. The next category was vacuuming, made up all newborns delivered by vacuum extraction. Newborns delivered through caesarean section $(\mathrm{C} / \mathrm{S})$ were also categorized as a single group. Apgar score at 5 minutes was categorized in three groups; 0-3, 4-7 and above 7. Newborns' gestational age was classified as pre-term ( $<36$ weeks), term ( $>36$ weeks) or not stated. The diagnosis was also classified into eight groups. The most prevalent conditions were prematurity, respiratory distress, infections, congenital anomalies, neonatal jaundice, sepsis and birth asphyxia. The age as of the time of admission was divided into four groups; 1 day, 2-7 days, 8-14 days and $15-28$ days 


\section{Statistical analysis}

A preliminary statistical analysis was done to examine the frequency distribution of the variables and cross tabulation of the variables with outcome. Univariate analysis was done to examine the variables associated with neonatal death. These variables which were significant in the univariate analysis were consequently included in the logistic regression model. The logistic regression model was used to determine the strength of association between these predictors and the outcome while adjusting for confounders. Confidence interval graph based on sum contrast ${ }^{9}$ from the logistic model was constructed to illustrate the percent of neonatal deaths for each predictor. The normal quantile-quantile (Q-Q) plot was used to verify the normality assumption of the residuals from the model. The plot of observed count versus expected count of neonatal deaths was used to evaluate the fit of the model.

\section{Ethical approval}

This study was approved by the Research and Development Unit (R\&D) of KATH and the Committee on Human Research, Publications and Ethics, Kwame Nkrumah University of Science and Technology, School of Medical Sciences and KATH, Kumasi on with reference number CHRPE/AP/365/15.

\section{Results}

Information on 5,195 newborns was used for the study. More than half of the newborns were males (55\%). The percentage of neonatal mortality among males was $20 \%$ and that of females was $20.5 \%$ (Table 1). More than $80 \%$ (888) of neonatal deaths occurred in the first day of life with $17.1 \%$ mortality. Out of the 5,195 newborns admitted to the unit, 3,022 (58.2\%) were delivered at the KATH while 2,173 (41.8\%) were delivered and referred from outside the KATH. There were 1,053 neonatal deaths over the period. representing a percentage mortality of 20.3. Table 1 shows the distribution of the various variables and proportion of neonatal death. 
Table 1. Distribution of neonatal characteristics

\begin{tabular}{|c|c|c|c|}
\hline Variables & Survived (\%) & Died (\%) & p-value \\
\hline$\overline{\text { Gender }}$ & & & 0.7186 \\
\hline Female & $1,860(79.5)$ & $480(20.5)$ & \\
\hline Male & $2282(80.0)$ & $573(20.0)$ & \\
\hline Birth Weight & & & $<0.005$ \\
\hline VLBW & $431(50.9)$ & $416(49.1)$ & \\
\hline LBW & $1,029(85.3)$ & $178(14.7)$ & \\
\hline Normal & $2682(85.4)$ & $459(14.6)$ & \\
\hline 5 Minute Apgar score & & & $<0.005$ \\
\hline $0-3$ & $66(32.7)$ & $136(67.3)$ & \\
\hline $4-7$ & $644(67.3)$ & $313(32.7)$ & \\
\hline $8-10$ & $3,432(85.0)$ & $604(15)$ & \\
\hline Gestational age & & & $<0.005$ \\
\hline Term & $963(91.7)$ & $87(8.3)$ & \\
\hline Preterm & $487(68.5)$ & $224(31.5)$ & \\
\hline Not Stated & $2,692(78.4)$ & $742(21.6)$ & \\
\hline Delivery Mode & & & $<0.005$ \\
\hline SVD & $2,358(76.2)$ & $736(23.8)$ & \\
\hline $\mathrm{C} / \mathrm{S}$ & $1,736(84.8)$ & $311(15.2)$ & \\
\hline Vacuum & $48(88.9)$ & $6(11.1)$ & \\
\hline Place of Delivery & & & $<0.005$ \\
\hline КАТН & $2,573(85.1)$ & 449 (14.9) & \\
\hline Referred & $1,569(72.2)$ & $604(27.8)$ & \\
\hline Admission age & & & $<0.005$ \\
\hline 1 day & $3,210(78.3)$ & $888(21.7)$ & \\
\hline 2-7 days & $728(85.1)$ & $127(14.9)$ & \\
\hline $8-14$ days & $102(86.4)$ & $16(13.6)$ & \\
\hline $15-28$ days & $102(82.3)$ & $22(17.7)$ & \\
\hline Diagnosis & & & $<0.005$ \\
\hline Prematurity & $888(69.1)$ & 397 (30.9) & \\
\hline Respiratory Distress & $208(72.2)$ & $80(27.8)$ & \\
\hline Infections & $1,120(91.7)$ & $102(8.3)$ & \\
\hline Congenital anomalies & $377(81.1)$ & $88(18.9)$ & \\
\hline Neonatal Jaundice & $799(91.8)$ & $71(8.2)$ & \\
\hline Birth asphyxia & $638(67.5)$ & $307(32.5)$ & \\
\hline Others & $112((93.3)$ & $8(6.7)$ & \\
\hline
\end{tabular}

From the univariate analysis there was no significant association of sex with neonatal mortality. Thus, it was excluded from the multivariate analysis. Other variables such as birth weight, 5-minute Apgar score, gestational age, delivery mode, admission age and main diagnosis were all significantly associated with neonatal mortality. From the logistic regression analysis, birth weight, place of delivery, 5-minute Apgar score, gestation and main diagnosis remained significantly associated with neonatal death. The logistic regression analysis also showed that the association of neonatal mortality with admission age and delivery mode disappeared. Compared to newborns born with Apgar score 8 or higher, newborns born with 5-minute Apgar score 4- 7 and lower than 4 were more likely to have higher neonatal mortality of 2 and 8 folds, respectively. Very low birth weight newborns had higher mortality than normal weight newborns (7 times more). Newborns born pre-term had a 2 times increased in the risk of death higher than newborns with term born. Newborns referred to the neonatal unit had higher percent of mortality than newborns born at KATH. Crude and adjusted odds ratios (OR) for each variable are presented in Table 2 . 
Table 2. Crude and adjusted Odds ratio (OR) from the logistic regression analysis with $95 \%$ Confidence intervals.

\begin{tabular}{|c|c|c|c|}
\hline Variable & Crude OR (95\%) & Adjusted OR (95\% CI) & p-value \\
\hline$\overline{\operatorname{Sex}}$ & & & 0.693 \\
\hline Female & 1 & & \\
\hline Male & $0.97(0.92,1.03)$ & & \\
\hline Birth Weight & & & $<0.005$ \\
\hline Normal & 1 & 1 & \\
\hline LBW & $1.01(0.84,1.22)$ & $1.23(0.98,1.55)$ & \\
\hline VLBW & $5.64(4.77,6.66)$ & $7.24(5.40,9.69)$ & \\
\hline 5 Minute Apgar score & & & $<0.05$ \\
\hline $8-10$ & 1 & 1 & \\
\hline $4-7$ & $2.76(2.35,3.24)$ & $2.06(1.69,2.50)$ & \\
\hline $0-3$ & $11.71(8.62,15.91)$ & $8.27(5.81,11.79)$ & \\
\hline Gestational age & & & $<0.05$ \\
\hline Term & 1 & 1 & \\
\hline Preterm & $5.09(3.88,6.67)$ & $2.23(1.60,3.12)$ & \\
\hline Not Stated & $3.05(2.41,3.86)$ & $1.96(1.50,2.55)$ & \\
\hline Delivery Mode & & & 0.553 \\
\hline $\mathrm{C} / \mathrm{S}$ & 1 & & \\
\hline SVD & $1.74(1.51,2.01)$ & & \\
\hline Vacuum & $0.70(0.30,1.64)$ & & \\
\hline Place of Delivery & & & $<0.05$ \\
\hline KATH & 1 & 1 & \\
\hline Referred & $2.21(1.92,2.53)$ & $2.40(2.00,2.87)$ & \\
\hline Admission age & & & 0.444 \\
\hline 1 day & 1 & & \\
\hline 2-7 days & $0.63(0.51,0.77)$ & & \\
\hline 8-14 days & $0.57(0.33,0.97)$ & & \\
\hline $15-28$ days & $0.78(0.49,1.24)$ & & \\
\hline Diagnosis & & & $<0.05$ \\
\hline Others & 1 & 1 & \\
\hline Prematurity & $6.26(3.03,12.95)$ & $1.02(0.47,2.13)$ & \\
\hline Respiratory distress & $5.38(2.51,11.44)$ & $4.00(1.82,8.79)$ & \\
\hline Infections & $1.28(0.61,2.89)$ & $0.84(0.39,1.81)$ & \\
\hline Congenital anomalies & $3.27(1.54,6.94)$ & $2.11(0.97,4.57)$ & \\
\hline Neonatal Jaundice & $1.24(0.58,2.65)$ & $0.70(0.32,1.54)$ & \\
\hline Birth asphyxia & $6.74(3.25,13.98)$ & $3.17(1.49,6.74)$ & \\
\hline
\end{tabular}

The observed and adjusted proportions of variables that were significant from the multivariate analysis are presented in Figure.1 together with the overall mean percent- age mortality. Newborns with birth asphyxia, congenital anomalies, infections and respiratory distress had higher neonatal mortality than the overall mean. The horizontal line indicates the overall mean. 


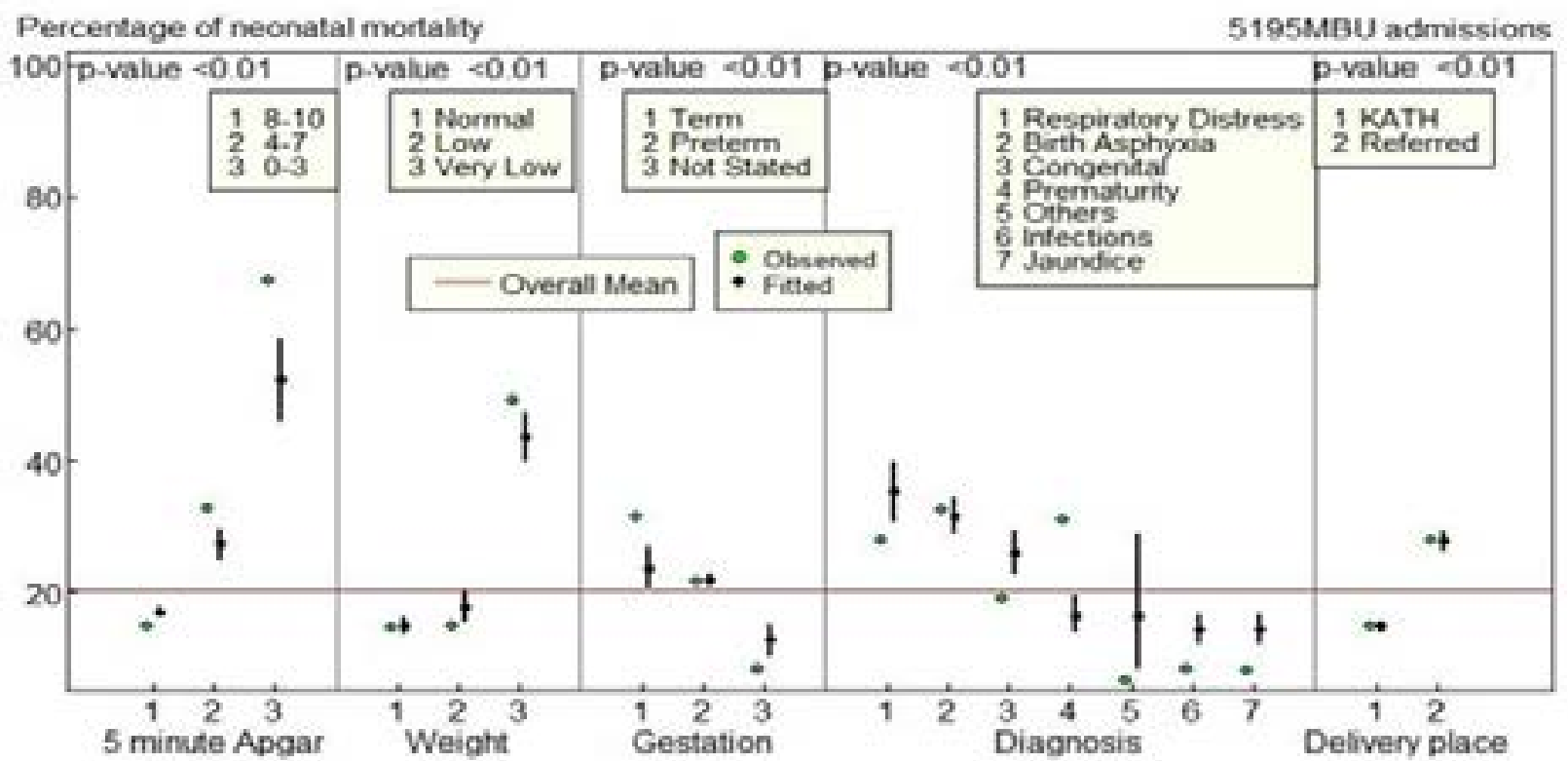

Figure 1: Mortality rate of each variable used in the logistic regression model

Quantile-Quantile (Q-Q) plot (Figure 2) of the residuals from the logistic regression model indicates that the model has provided an acceptable fit for the outcome variable (neonatal mortality). It also shows that the estimates from the model are asymptotically normal. A plot of the observed and fitted values is also provided in figure 3. The values match each other in a vertical pattern.

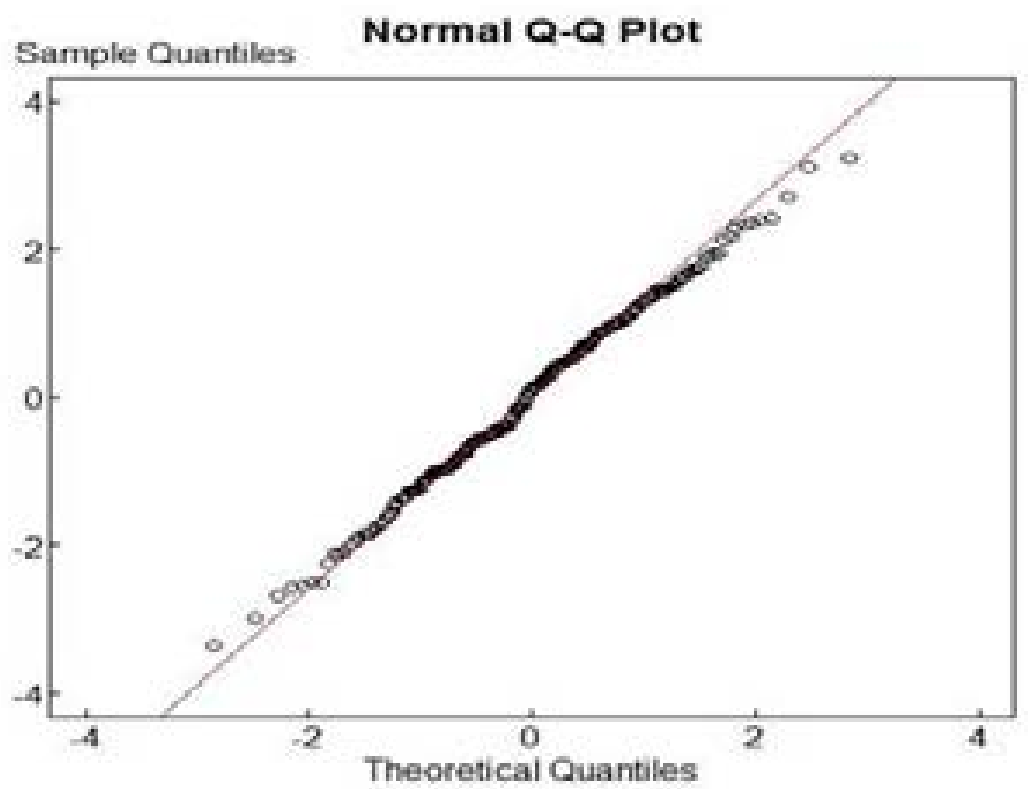

Figure 2: Normal quantile-quantile plot of residuals 


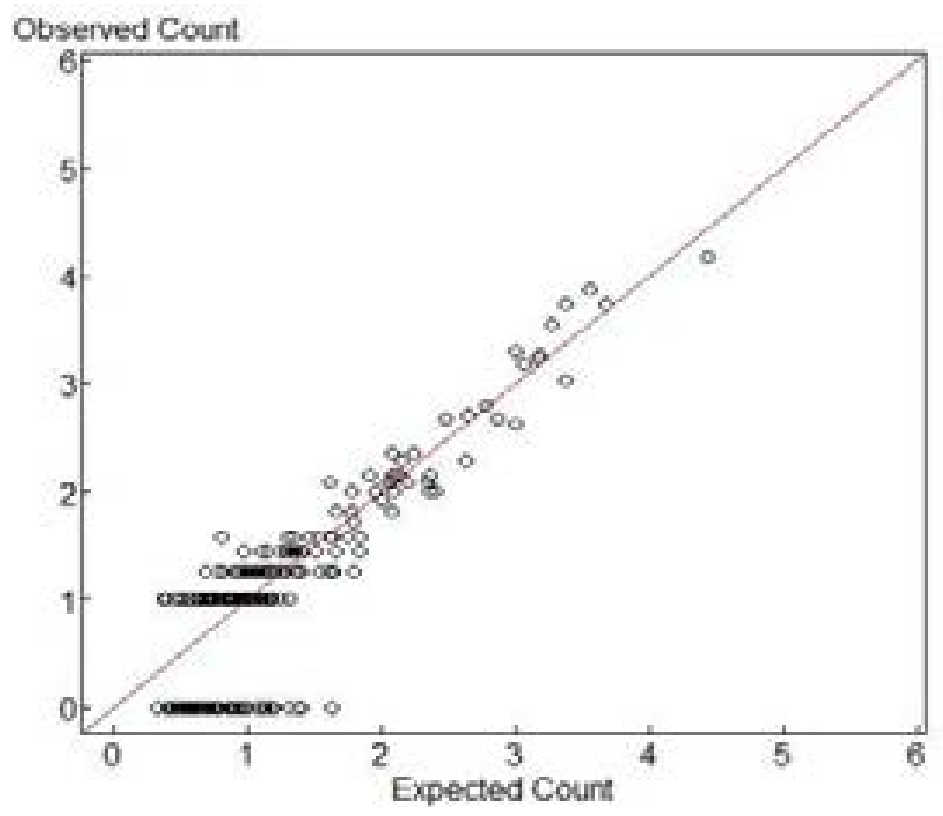

Figure 3: Expected and observed values

\section{Discussion}

Our study revealed that the survival of a neonate in the neonatal unit depended on the birth weight, 5-minute Apgar score, place of delivery, gestational age and discharge diagnosis. Other population and hospital based studies conducted in Pakistan and the Korle-Bu Teaching Hospital in Ghana have found these factors to be associated with neonatal death ${ }^{10,11}$. Although different researches have documented evidence to show higher mortality percentages in male than female infants ${ }^{12,13}$, our study found otherwise. We found no significant difference in the mortality of males and females. However, the reasons for such contrast result could not be explained from the result. The percentage of neonatal death for newborns delivered at KATH was $15 \%$ while that of newborns referred from other health facilities was $27.6 \%$ The overall mortality at the neonatal unit was $20.3 \%$. This result is lower than $29.1 \%$ and $22.4 \%$ that was recorded for the neonatal intensive care unit of the Cairo university children's hospital, Egypt and Abha Hospital, Saudi Arabia $^{13,14}$. However, percentage of mortality among newborns referred to the facility still remains very high. This is because most newborns referred to KATH are usually very sick and require specialist care that is not provided by primary health facilities.
Although gestational age was a significant risk factor, it was only significant among newborns delivered at KATH. Pre-term birth was associated with a higher risk of death and higher mortality percentage more than the overall percentage. This is comparable with results presented by $\mathrm{Lawn}^{3}$ and $\mathrm{Hsu}^{6}$. Pre-term newborns require special care from skilled workers to improve their chances of survival. Such special care involves providing warmth and feeding support ${ }^{4}$. The major conditions associated with neonatal death were infections, respiratory distress, prematurity, neonatal jaundice, sepsis, infections birth asphyxia and other neonatal conditions. Recent population and hospital based studies have also corroborated these findings ${ }^{10,15,16}$. Among these neonatal conditions, birth asphyxia recorded the highest death rate. Clean birth practices have shown to significantly reduce neonatal mortality. Basic well known hygienic practices such as hand washing and maintaining a clean environment are poorly observed $^{4}$. In this present study birth weight was a major risk factor of neonatal death. The results revealed that VLBW had higher risk of neonatal mortality than newborns with normal weight. Other studies in sub-Saharan Africa ${ }^{17,18}$ and Asia $^{14}$ have made similar findings to the effect that birth weight is a significant risk factor of neonatal mortality. However, the odds ratio of death for low birth weight infants is lower than what was reported in 
a five-year study conducted at the facility in $2012^{19}$. This shows that the MBU of the KATH has made great strides in saving low birth weight infants.

\section{Study limitation}

This was a hospital based study and not a representation of the entire population in the city. Although the annual deliveries figure for the hospital exceed 10,000, this study focused only on sick neonates. Data for this study is retrospective and thus, there were cases of missing information although not significant.

\section{Conclusion}

The neonatal mortality percentage at the neonatal unit of the KATH is still high. This study found that newborns referred from home or other health facilities, newborns having very low birth weight, 5 minute Apgar score of less than 4, preterm birth and having congenital anomalies were more likely to have a higher percentage. There is, therefore, the need for continuous attention and strengthening of new-born interventions to help reduce the risk reduce the risk of mortality among neonates delivered at $\mathrm{KATH}$

\section{Acknowledgement}

Our tremendous thanks go to Emeritus Prof. Dr. Donald McNeil for his comments and support during the conduct of the study and to the Mother and Baby Unit, KATH for providing the data on neonatal admissions.

\section{Funding}

This work was supported by the Higher Education Research Promotion and the Thailand's Education Hub for Southern Region of ASEAN Countries Project Office of the higher Education Commission.

\section{Conflicts of interest}

We certify that there is no conflict of interest with any financial organization regarding the material discussed in this article.

\section{References}

1. Shiffman J. Network advocacy and the emergence of global attention to newborn survival. Health Policy Plan 2015; $1-13$
2. Akter T, Dawson A, Sibbritt D. What impact do essential newborn care practices have on neonatal mortality inlower-middle income countries? Evidence from Bangladesh. J Perinatol 2016; 36(3):225-230. PubMed

3. Lawn JE, Blencowe H, Oza S, You D, Lee ACC, Waiswa $P$ et al. Every Newborn: progress, priorities, and potential beyond survival. Lancet. 2014;384(9938):189-205

4. Lawn JE, Davidge R, Paul VK, Costello A, Kinney MV, Segre J. et al. Born Too Soon: Care for the pre-term baby. Reprod Health 2013;10(Suppl 1): S5

5. World Health Organization. 2012. Committing to Child Survival: A Promise Renewed. Progress Report. Available from: http://apromiserenewed.org

6. Hsu S-T, Hsieh C-J, Chen H-W, et al. Nationwide Birth Weight and Gestational Age-specific Neonatal Mortality Rate in Taiwan. Pediatr Neonatol. 2014;56(3):149-158

7. Ministry of Health $(\mathrm{MoH})$, Ghana Health Service (GHS). 2014. Ghana National Newborn Health Strategy and Action Plan 2014-2018.

8. World Health Organization. 2016. Global Health Observatory Data. Available from Available from:https:// www.tradingeconomics.com/ghana/mortality-rate-neonatal-per-1-000-live-births-wb-data.html

9. Tongkumchum P, Mcneil D. Confidence intervals using contrasts for regression model. Songklanakarin J Sci Technol. 2009;31(2):151-156

10. Welbeck J, Biritwum RB, Mensah G. Factors affecting the survival of the "at risk" newborn at Korle Bu Teaching Hospital, Accra, Ghana. West Afr J Med. 2003;22(1):55-58 11. Jehan I, Harris H, Salat S, Zeb A, Mobeen N, Pasha O. et al. Neonatal mortality, risk factors and causes: a prospective population-based cohort study in urban Pakistan. Bull World Health Organ. 2009;87(2):130-138

12. Anand K, Kant S, Kumar G, et al. Neonatal morbidity and mortality of sick newborns admitted in a teaching hospital of Uttarakhand. CHRISMED J Heal Res. 2014;1(4):247-253

13. Seoud I, El-din RMG, Said RN, Hessin HA. Predictors of Neonatal Mortality in Intensive Care Unit in Children's Hospital. Alexandria J Pediatr. 2005;19(1):93-97.

14. Arafa MA, Alshehri MA. Predictors of neonatal mortality in the intensive care unit in Abha, Saudi Arabia. Saudi Med J. 2003;24(12):1374-1376. PubMed

15. Ghana Statistical Service (GSS), Ghana Health Service (GHS), and ICF Macro. 2015. Ghana Demographic 
and Health Survey 2014: Key Findings. Retrieved from http://www.measuredhs.com/pubs/pdf

16. Edmond KM, Quigley M a, Zandoh C, et al. Aetiology of stillbirths and neonatal deaths in rural Ghana: implications for health programming in developing countries. Paediatr Perinat Epidemiol. 2008;22(5):430-437. PubMed 17. Ajaari J, Masanja H, Weiner R. Impact of Place of Delivery on Neonatal Mortality in Rural Tanzania. Int J MCH AIDS. 2012;1(1):49-59. PubMed
18. Adetola AO, Tongo OO, Orimadegun AE, Osinusi K. Neonatal mortality in an urban population in Ibadan, Nigeria. Pediatr Neonatol. 2011;52(5):243-250. PubMed 19. Siakwa M, Kpikpitse D, Laryea T, Ankobil A, Dare S, Ebu N. A five-year neonatal mortality trend in a Ghanaian Teaching Hospital after the implementation of strategies to achieve the Millenium Development Goal (MDG) 4. Int J Paediatr Child Heal. 2014;2(1):43-49 